\title{
Functions and Implications of Circular RNAs in Antiviral Immunity
}

\author{
Zubaida Hassan \\ Department of Microbiology, School of Life Sciences, Modibbo Adama University of Technology, Yola, Nigeria \\ Email: zubaidahassan@mautech.edu.ng
}

How to cite this paper: Hassan, Z. (2019) Functions and Implications of Circular RNAs in Antiviral Immunity. Advances in Microbiology, 9, 602-615.

https://doi.org/10.4236/aim.2019.97037

Received: April 3, 2019

Accepted: July 19, 2019

Published: July 22, 2019

Copyright $\odot 2019$ by author(s) and Scientific Research Publishing Inc. This work is licensed under the Creative Commons Attribution International License (CC BY 4.0).

http://creativecommons.org/licenses/by/4.0/

\begin{abstract}
In addition to mature mRNAs, splicing of pol II transcripts generates numerous other RNAs including circular RNAs (circRNAs). circRNAs are a group of transcripts generated by non-sequential back splicing or forward splicing of exons, introns or a combination of both from a donor to an acceptor target. This forms covalently closed RNA molecule i.e. without free 5' end caps or 3' Poly (A) tails thereby making them more stable than their linear counterparts. Though they are recognized as part of non-coding RNAs, long noncoding precisely; evidence of translations has been reported from these molecules. circRNAs are considered emerging new members of the gene regulatory family. These circRNAs have several potential modes of action, some of which serve as microRNAs sponges shown in Table 1, protein binding RNAs, cellular transports, transcriptional regulators and modulating immune system. Viral circRNAs may evade immune recognition by many proposed mechanisms. These include undergoing slicing by host splicing machinery, being single stranded lacking free 5' and 3' ends, having miRNA sponge function, convenient regulatory functions which include initiating lytic replication, etc. Pattern of expression of these circRNAs differs from healthy individuals to different stages of viral infectious diseases. Thus, their use as potential biomarkers for diagnostics and future therapeutics targets have been proposed, especially with regards to viral infections that seem impossible for the human immune system to totally eliminate like the EpsteinBarr viral infection. So, this review summarizes the functions and implications of circRNAs regarding antiviral immunity.
\end{abstract}

\section{Keywords}

circRNAs, Antiviral Immunity, Biomarker

\section{Introduction}

Antiviral immunity in a cell is any form of innate immunity that renders the cell 
directly non-permissive to particular group of viruses by restricting any step in the viral replication and assembly [1]. Pattern recognition receptors are the most widely known and studied antiviral mechanisms where viral infection is inhibited indirectly by inducing interferons and other antiviral molecules [1]. Antiviral response can be triggered posttranscriptionally via sequence-specific mechanisms. For decades, RNA interference (RNAi) has been reported to be important in sequence-specific gene silencing mechanisms [2], this has been extensively studied genetically with respect to both endogenously (miRNA) and synthetically (siRNA) generated RNA molecules. Recently, studies on circRNAs of both cellular and viral origins are gaining attention and some of which are associated with posttranscriptional antiviral immunity.

Table 1. Summary of some functions associated with circRNAs.

\begin{tabular}{|c|c|c|c|c|}
\hline $\mathrm{S} / \mathrm{N}$ & circRNA & Function & Mechanism & Reference \\
\hline 1 & circRNA & Translation potential & From their parental genes & $\begin{array}{l}\text { [21] Pamudurti et al., 2017; } \\
\text { [8] Ungerleider et al., } 2018\end{array}$ \\
\hline 2 & $\begin{array}{l}\text { Endogenous translatable } \\
\text { circRNAs [30] [30] }\end{array}$ & Translation potential & Associated with polysome & [30] Yang et al., 2017 \\
\hline 3 & has_circ_0041407 & Translation potential & Internal ribosome entry site (IRES) & [30] Yang et al., 2017 \\
\hline 4 & circ-ZNF609 & $\begin{array}{l}\text { Translated into } \\
\text { functional proteins }\end{array}$ & $\begin{array}{l}\text { Cap-independent and } \\
\text { splicing-dependent manner }\end{array}$ & [29] Legnini et al., 2017 \\
\hline 5 & circRNAs & modulating RNA splicing & & [21] Pamudurti et al., 2017 \\
\hline 6 & circRNAs & Regulation gene expression & $\begin{array}{l}\text { Transcriptional, post-transcriptional, } \\
\text { and translational }\end{array}$ & $\begin{array}{c}\text { [19] [37] Wang et al., 2017; } \\
\text { Zhang et al., } 2017\end{array}$ \\
\hline 7 & $\begin{array}{l}\text { CircBHLF1 } \\
\text { and circBHLF1alt }\end{array}$ & $\begin{array}{l}\text { Activation of lytic } \\
\text { viral DNA replication }\end{array}$ & \multirow{6}{*}{$\begin{array}{l}\text { Facilitating } \mathrm{Cp} \text { initiated } \\
\text { transcript diversity }\end{array}$} & [8] Ungerleider et al., 2018 \\
\hline \multirow{3}{*}{8} & circLMP2 & EBV reactivation & & \\
\hline & circRPMS1 & $\begin{array}{l}\text { Supporting EBV tumour } \\
\text { phenotype in stomach cancer }\end{array}$ & & [8] Ungerleider et al., 2018 \\
\hline & circEBNA & Associated with type III latency & & \\
\hline \multirow[t]{2}{*}{9} & ciRS-7 & \multirow[t]{2}{*}{ miRNAinhibitor } & & $\begin{array}{l}\text { [38] [39] Peng et al., 2015; } \\
\text { Hansen et al., } 2013\end{array}$ \\
\hline & circBART & & & [7] Toptan et al., 2018 \\
\hline 10 & circ-Foxo3 & Blocks cell cycle & \multirow[t]{3}{*}{$\begin{array}{l}\text { Formation of circ-Foxo3/CDK2/p21 } \\
\text { complex that prevent interaction } \\
\text { between p } 21 \text { and cyclin A and that } \\
\text { between CDK2 with cyclin E and } \\
\text { p27 as such block transition of } \\
\text { cell cycle from G1 to } S \text { phase }\end{array}$} & $\begin{array}{c}\text { [40], [41] } \\
\text { Fischer \& Leung, 2017; } \\
\text { Du et al., } 2017\end{array}$ \\
\hline 11 & circHIPK3 & Blocks cell cycle & & [42] Zheng et al., 2016 \\
\hline 12 & Circ-Foxo3 & $\begin{array}{l}\text { Interfere with subcellular } \\
\text { localisation of proteins }\end{array}$ & & [40] Fischer \& Leung, 2017 \\
\hline 13 & $\begin{array}{c}\text { Purified circRNAs } \\
\text { (Only circRNA } \\
\text { generated by } \\
\text { self-splicing and } \\
\text { not with spliceosome) }\end{array}$ & $\begin{array}{l}\text { Confer protection } \\
\text { against viral infection }\end{array}$ & $\begin{array}{l}\text { Activates RIG-I and by } \\
\text { offsetting the effects of miRNAs } \\
\text { on target gene expression }\end{array}$ & $\begin{array}{l}\text { [26] Chen et al., } 2017 \\
\text { [19] Wang et al., } 2017\end{array}$ \\
\hline
\end{tabular}


Circular RNAs (circRNAs) have a covalently closed loop feature i.e. without 5' caps or 3' Poly (A) tails [3] generated by non-sequential back splicing [4] [5] [6] [7] or forward splicing [8] of exons, introns or a combination of both. They are highly stable (due to their nuclease resistance properties) [9], evolutionarily conserved across species [10] and are used by viruses to modify the host cells' environment in ways that support of the virus' needs without eliciting immune clearance [8]. Initially, circRNAs were regarded as viral genomes or by-products of pre-mRNA alternative splicing, [11]. Their widespread abundance in eukaryotic cells suggests that circRNAs are not simply accidental by-products of missplicing events, but represent an indispensable part of non-coding RNAs (ncRNAs) families [12].

With recent developments in high-throughput technologies such as high tech, RNAse $\mathrm{R}$ protected sequencing and microarray techniques among others, enabled scientists to conduct comprehensive analyses on the abundance, composition, expression profile and molecular function of these circRNAs [13]. So far, large amounts of circRNAs have been successfully identified in a variety of organisms including plants [14], animals, [15], [16], humans [17] and viruses [7], [8].

ncRNAs play important roles in controlling gene expression at both transcriptional and post-transcriptional levels. Mammalian genomic DNA consists of more than $62 \%$ transcription template. However, less than $2 \%$ actually codes for protein. This indicates abundance of ncRNAs in human transcriptome [18], [19]. CircRNAs were reported to act as miRNA sponges with regulatory function in gene expression [20], modulating RNA splicing [21], scaffolds for the assembly of protein complexes, templates for translation, and modulators of rRNA and tRNA biogenesis [19]. Viruses are such unique organisms that cannot even be able to encode machineries required for their survival, so if they produce any substance, it is likely to have advantages to their existence. CircRNAs are produced in large quantity by viruses like EBV for example. This puzzled virologists to focus on this novel transcript in trying to find out if it is utilized in their ability to establish lifelong infection in humans as well as cell transformation.

It has been shown that circRNAs are an association with the occurrence and development of human diseases, for example, cutaneous squamous cell carcinoma [22], chronic thromboembolic pulmonary hypertension [23], type 2 diabetes mellitus [24], osteoarthritis, cardiovascular and neurological diseases, aging and cellular senescence [25]. Therefore, these molecules are considered as potential diagnostic biomarkers and therapeutic targets [19]. CircRNAs can be used to boost immune activation especially for antiviral therapeutic purposes. For instance, in host-virus interaction, viral circRNAs are recognised by the nucleic acid sensor RIG-I which triggers innate immune signaling [26] by activation of RBP factors NF90/NF110 which modulate circRNA biosynthesis and suppress viral infection [27] and thus provide protection against viral infection. CircRNAs have also been shown to be implicated in immune pathways such as modulation of B cell activation and antigen-presentation process [28]. 
However, a successful virus such as EBV that establish lifelong latency in over $90 \%$ of immunocompetent individuals produce a huge number of these circRNAs in all stages of its life cycle [7], [8]. So, several intriguing questions on how this group of transcript interplay and interact with host immune system need to be clarified. Therefore, this review summarises the functions and implications of circRNAs with regards to antiviral immunity.

\section{Protein-Coding Ability of CircRNAs}

Despite being ncRNAs, circRNAs have the potentials of protein coding since quite a number of them are made up of exon from pre-mRNA transcripts. It was reported that smaller peptide derivatives can be made from circRNAs derived from their parental genes [8], [21]. From the study by Legnini et al., [29], circRNA, circ-ZNF609-a 753-nt contains open reading frame (ORF) and can be translated into functional proteins in a cap-independent and splicing-dependent manner. Some circRNAs were found to be able to interact with cap-independent translation factors such as eukaryotic initiation factor 3 (eIF3) and N6-Methyladenosine (m6A), suggesting the translational potentials of these circRNAs [30]. Yang et al., [30] also revealed that has_circ_0041407 contains an internal ribosome entry site (IRES) element and can be translated into a $31-\mathrm{kDa}$ chimeric protein. The presence of IRES sequences drive circRNA translation [19] as it is an important factor in the initiation of cap-independent translation.

Of the hundreds of endogenous translatable circRNAs found associated with polysome, many contain m6A sites. Suggesting a general function of circRNA in the translation landscape of human genome [30]. A minigene reporter containing split GFP was constructed to demonstrate that a circRNA can be translated using a viral IRES [31]. All circRNAs (including negative controls) containing several other endogenous IRES or control sequences in human genome efficiently initiated GFP translation. However, this translation from the circRNA was eliminated when the sequence of restriction sites was left between the stop and start codons of GFP in the circRNA reporter. This suggested that IRES is serving to facilitate translation initiation in circRNAs [30]. Sequence analysis of all the negative controls were found to contain RRACH fragment $(R=G$ or $A ; H$ $=\mathrm{A}, \mathrm{C}$ or $\mathrm{U}$ ) near the start codon [30], resembling the consensus motif of $\mathrm{m} 6 \mathrm{~A}$ modification (i.e., RRACH motif), the most abundant internal modification of RNAs [32], [33]. Compared to all coding mRNAs, the putative m6A motifs are significantly enriched in known circRNAs [30].

Modestly reduced protein translation was observed from circRNA when eIF3A, (-eIF3 subunit bound to viral IRES) was depleted but the depletion did not affect linear mRNA translation [34]. It was reported by Meyer et al. [35] that eIF3A is involved in the m6A-promoted translation. CircRNA translation was hypothesized to be initiated through a mechanism fundamentally different from linear mRNA that is initiated by ribosomal scanning [30]. Therefore, the focus 
was directed to cap-independent translation where a non-canonical eIF4G protein (eIF4G2) directly recognizes an IRES to initiate eIF4 complex assembly in the absence of eIF4E leading to translation initiation [36]. When eIF4G2 was depleted, protein translation from circRNA was significantly reduced but it had no effect on translation from linear mRNA [30].

Viral circRNAs were reported to be expressed from small, well-defined operons [7], [8] which interact with ribosomes [8] suggesting translation potentials. These small peptides synthesized may have significant impact on the viral survival since the transcripts are produced in large quantity. It was reported that EBV circRNAs were expressed at levels that are comparable to or higher than the majority of cellular encoded circRNAs supporting the contention of potential functional relevance [8]. Could these circRNAs contribute to EBV tumorigenesis or antiviral immunity? Utilizing ncRNAs to modulate host cell signaling pathways was speculated to be an important viral strategy for moulding the host cell environment in ways that support of the virus' needs without eliciting immune clearance [8]. If circRNAs are confirmed to be vital for antiviral immunity, they can serve as suitable substrate for targeted therapies that may be more specific and less toxic than the currently available therapies [7].

\section{Cellular Transport and Function of CircRNAs}

Although circRNAs were proposed and reported to act as miRNA sponges, that is regulatory function in gene expression [20] mainly due to their origin from the same locus that encodes miRNAs, this finding may not always hold true since a lot of other circRNAs have been reported from protein coding loci and were to some extend thought to be translated into small peptides with functions not necessarily similar to their parental loci proteins. For example, EBV cicrRNAs were reported from four different loci including EBNA and LMP loci which are protein coding regions [8]. circRNAs function well in modulating RNA splicing [21], promote the assembly of protein complexes, act as templates for translation, and modulators of rRNA and tRNA biogenesis [19]. Therefore, it is suggested that gene expressions are regulated by circRNAs at transcriptional, post-transcriptional, and translational levels [37].

CircBHLF1 and circBHLF1alt because of the cis proximity of their originating locus to ori-Lyt were hypothesized to provide unique molecular features that engage with the replication complex and regulate lytic viral DNA replication due to their closed circular structure. Despite originating from miRNA locus, they were dissociated from sponging function [8]. CircBARTs are another group of EBV circRNAs that arise from the same gene locus encoding the miRNAs and were found not to be associated with polysome indicating non translational function [7] and so, suggesting regulatory function rather than protein coding.

Linear LMP2B transcripts or its promoter derive the initiation and expression of circLMP2. CircLMP2 back-splicing may be regulated by cis and trans acting mechanisms that support a potential unique role for circLMP2 in reactivation 
[8]. Others like CircRPMS1 were found to be expressed in EBV positive stomach cancer supporting in vivo relevance and possible roles in supporting the tumour phenotype while circEBNA were associated with type III latency specific processes such as facilitating $\mathrm{Cp}$ initiated transcript diversity [8].

Molecular sponge function of circRNAs was demonstrated by [38], [39] when they showed that ciRS-7 acts as an endogenous miR-7 inhibitor and so counteract its effect. Similar function was proposed for EBV circBART because none of them were found associating with the polysome fraction despite some of the circBART are purely exogenic [7]. Therefore, viral circRNAs may play tremendous diverse function that could be vital in its ability to evade host competent immune system and establish persistent infection.

Ignoring cell cycle inhibition signals is a great mechanism of tumour cells, circRNAs have been found to facilitate interaction of protein complexes that blocks the cell cycle progression in response to cell overgrowth and thus promote proliferation. For example, circFoxo3 forms a ternary structure by interacting with cyclin-dependent kinase 2 (CDK2) proteins and CDK inhibitor p21. This circ-Foxo3/CDK2/p21 complex blocks the cell cycle by preventing the interaction between $\mathrm{p} 21$ and cyclin A and that between CDK2 with cyclin E and p27 as such block transition of cell cycle from G1 to S phase [40], [41]. Similarly, cellular growth been shown to be supressed with depletion of circHIPK3 in HEK293T cells [42].

Subcellular localisation of a protein is crucial for its function. CircRNAs have been predicted to be implicated in the transport of proteins, they bind to RNA Binding Protein (RBP) and interfere with their transport to specific subcellular locations [43]. Circ-Foxo3 (associated with cytoplasmic localisation) was reported to bind to proteins like inhibitor of differentiation-1 (ID-1), the transcription factor E2F1, hypoxia inducible factor- $1 \alpha$ (HIF- $1 \alpha$ ) and focal adhesion kinase (FAK), and prevent them from translocating to other locations [40]. HuR is a part of a regulatory $\mathrm{RBP}$ components reported to be interacting with circRNAs [44].

Other functions such as regulation of alternative splicing and gene expression, templates for translation [19] [31] [45] [46] were also reported in association with circRNAs. It was reported that production of circRNA may have consequences on the transcription of their linear transcripts [25]. In the Muscleblind (MBL) gene for example, circRNA MBL forms autoregulatory loop as it binds to the MBL gene itself [46] while in Arabidopsis, a circRNA derived from the SEPALLATA3 gene has pause in transcription of the linear mRNA by forming R-loop at its cognate DNA, and also affect alternative splicing through exon-skipping nascent transcript [47].

With increased stability associated with circRNA compared to linear RNAs, they are of great advantage as marker molecules especially in liquid biopsy markers [16] [45] and could potentially be used in predictive and/or clinical diagnosis [7] [8]. 


\section{CircRNAs Involved in Antiviral Immunity}

Innate immune response plays a significant role in early response to viral and other infections. Viral infections are recognised through variety of pattern recognition receptors (PRRs) which include Toll-like receptor 3 (TLR3), retinoic acid-inducible gene-I (RIG-I), melanoma differentiation-associated gene 5 (MDA5) and laboratory of genetics, physiology 2 (LGP2) [48], [49] and oligoadenylate synthetase-like (OASL) [25]. It was shown that purified circRNAs activates RIG-I and confers effective immune protection against viral infection [26]. During Venezuelan equine encephalitis virus infection, a 10-fold decrease in the expression of RIGI and OASL were observed. However, when HeLa cells were transfected with circRNA, upregulation of these PRRs (up to 500-fold and 200-fold, respectively) were recorded [26].

However, [19] indicated that RIG-I is activated by circRNAs made with self-splicing introns but not with the same circRNAs created with endogenous introns and spliced by cellular splicing machinery. Suggesting unknown RIG-I-regulatory function associated with endogenous spliced circRNAs. Viruses entirely depend on host machineries to generate and process their transcripts. This could explain why viral circRNAs do not activate RIG-I and possible other PRRs to protect the virus against immune recognition. Another possible reason could be because RIG-I and MDA5 are cytoplasmic RNA helicases that bind and destroy dsRNA [50]. EBV can evade RIG-I activity since all the EBV circRNAs reported were ssRNAs. It is yet unclear how circRNAs interact with RIG-I despite having no 5' or 3' ends.

One mechanism proposed by [19] suggests that these circRNAs may effectively induce antiviral immune responses via offsetting the effects of miRNAs on target gene expression. Cellular miRNAs are crucial in immune regulation and viral pathogenesis [51] [52] [53]. EBV and a number of other viruses manipulate these miRNAs to suit their needs [54] by encoding their own endogenous miRNA [55]. It is well known that viruses can modulate cellular processes such as apoptosis-related and anti-tumour pathways [56] [57], inhibit the expression of viral genes in other to protect virus-infected cells from the host immune surveillance [58].

Although it is still at infancy, computational analyses have started to pinpoint some of the role played by circRNA in antiviral immunity. For instance, a human circRNA, hsa_circ_002048, derived from ANKRD11 transcript harbours 36 binding sites for EBV-encoded miR-BART20-5p and participate in the cellular pathways that are associated with viral entry, replication as well as virulence [8], [19]. miR-BART20-5p was reported to directly target EBV immediate early genes like $B R L F 1$ so, have a crucial role in maintenance of latency in EBV-associated tumors [59]. It is not surprising that circBARTs enhance this mechanism because many EBV-derived circRNA are circBARTs that arise from the same gene locus encoding the EBV miRNAs [7] [60].

In a study of avian leukosis virus subgroup (ALV-J), circRNAs upregulated in 
ALV-J resistant chickens were found to be targeting miRNAs implicated in modulation of B cell activation and antigen-presentation process [28]. Similarly, miRNAs found binding to differentially expressed circRNAs in Grass carp reovirus (GCRV) serve important functions in hemostasis immune responses [61]. CircPVT1 was found to sponge the functions of miR-125 in gastric cancer [62] including antiviral immunological defence. Since these circRNAs are resistant to nuclease activities due to their closed circular structure, association of circBHLF1 and circBHLF1alt with ori-Lyt might explain appearance of EBV lytic phase in healthy individuals at time interval. Nonetheless, what signal is required for these circRNAs to turn on the expression of the lytic replication?

\section{Uses as Biomarkers and Therapeutic Targets}

The differential expression patterns and characteristics of circRNAs between diseased and normal cell [17], [42], [63], coupled with their abundant detection in human blood [64], [65], saliva [66], gastric fluid [67] and exosomes [68] make them ideal candidates as biomarkers. Disease-relevant information provided by circRNA may not be revealed by canonical RNA analysis [37]. For example, hsa_circ_002059 was found to be significantly down-regulated in plasma of gastric cancer post-operative patients compared to pre-operative patients [69]. This low expression was correlated with tumour size, distant metastasis and TNM stage of the cancer [69], [70].

CircPVT1 is another circRNA found differentially (elevate) expressed in gastric cancer tissues [62]. CircPVT1 is known to promote cell proliferation by acting as a sponge for miR-125. Members of miR-125 family were validated to be down-regulated in other to exhibit their known disease-suppressing properties in many different types of disease conditions and they are crucial in host immune defence against bacterial or viral infections [71]. Thus, circPVT1 is thought to be an independent prognostic marker for the overall survival and disease-free survival time of gastric cancer patients [37].

Due to their abundance, Epstein-Barr encoded RNAs (EBERs) expression in EBV infection, EBERs are used in both research and diagnostic laboratories as biomarkers for EBV. In the current times, EBV circRNAs are found to reveal EBV infection (with as low as $<0.05 \mathrm{EBV}$ genome copy number per cell) which happen to be undetectable with EBERs markers [7]. All EBER-positive PLTDs tested were found to be positive for EBV circBART RNA, however, 3 EBER-negative PTLD tissues had weak EBV circBART RNA. When retested, these samples had EBV genome copy numbers comparable to EBV-positive, suggesting false-negative results for the EBER diagnostic test [7].

The circRNAs are highly resistant to exonuclease degradation and may be retained even in formalin-fixed, paraffin-embedded specimens. They constitute large part of eukaryotic transcripts and show certain levels of tissue and cell specificity [37]. Cellular circRNAs that are induced in various tumour types have shown promise as biomarkers for some cancers [10], [37]. Presence of vi- 
rus-encoded circRNA might have even better discriminatory power for use as new diagnostic tests to detect these viruses [7].

Targeting circRNAs was suggested to be more promising therapeutic effect against viral infection. By acting as miRNA sponges, circRNAs are implicated in the initiation and progression of human diseases. It was reported by Wang et al. [19], that circRNAs have the ability to suppress the function of the cellular or viral miRNAs. Similarly, circBHLF1 was reported to have a potential role in reactivation of EBV infection by facilitating lytic EBV replication. This is because the circBHLF1 was found in association with OriLyt in the nucleus of infected cell [8].

\section{Summary and Conclusions}

Recently, circRNAs have been shown to be part of total transcriptome of many organisms from mammals to lower organisms. The presence of circRNAs in viruses and their ability to interplay with host immune system is currently gaining attention as many studies are pointing towards their role in antiviral immunity. Formation of circRNAs is most often coupled with alternative splicing utilizing host splicing machinery, what circumstances will warrant preferences of circularisation of the transcript remains unknown. How is the circRNA generated using spliceosome complex differs in function (like activating RIG-I) from their counterpart generated by self-splicing? Could epigenetic marks play a role in formation and function of circRNAs? circRNAs are covalently closed loop feature i.e. without 5' caps or 3' Poly (A) tails generated by non-sequential back splicing or forward splicing of exons, introns or a combination of both.

Due to the fact that EBV circRNAs for example originate from different loci and exhibited diverse patterns of expression as well as different sub-cellular distributions, they have potentials for diverse function that could potentially define ability of the virus to establish successful lifelong latency in vast majority of human population worldwide. The studies of circRNAs are just coming up, focusing more on their potentials in antiviral immunity which will be of great revolution to the field of virology as it will both increase our knowledge on the viral life cycle and help in designing effective therapeutic strategies against many viral diseases including those known to cause lifelong latent infection in immunocompetent host.

\section{Literature Review Criteria}

Information for this review was compiled by searching Google and other academic databases like PubMed for published articles using search terms "circular RNA", "alternative splicing output" and "transcripts" + the terms "antiviral immunity", "function" or "emerging roles". Full articles were obtained, and references were checked for additional material where necessary. Information search skills coupled with knowledge in molecular biology, virology and immunology were utilized in the literature search process. 


\section{Conflicts of Interest}

The author declares no conflicts of interest regarding the publication of this paper.

\section{References}

[1] Yan, N. and Chen, Z.J. (2012) Intrinsic Antiviral Immunity. Nature Immunology, 13, 214-222. https://doi.org/10.1038/ni.2229

[2] Van Rij, R.P. and Andino, R. (2008) The Complex Interactions of Viruses and the RNAi Machinery: A Driving Force in Viral Evolution. In: Domingo, E., Parrish, C.R. and Holland, J.J., Eds., Origin and Evolution of Viruses, Elsevier, Amsterdam, 161-181. https://doi.org/10.1016/B978-0-12-374153-0.00007-2

[3] Liang, D. and Wilusz, J.E. (2014) Short Intronic Repeat Sequences Facilitate Circular RNA Production. Genes \& Development, 28, 2233-2247. https://doi.org/10.1101/gad.251926.114

[4] Starke, S., et al. (2015) Exon Circularization Requires Canonical Splice Signals. Cell Reports, 10, 103-111. https://doi.org/10.1016/j.celrep.2014.12.002

[5] Memczak, S., et al. (2013) Circular RNAs Are a Large Class of Animal RNAs with Regulatory Potency. Nature, 495, 333-338. https://doi.org/10.1038/nature11928

[6] Jeck, W.R. and Sharpless, N.E. (2014) Detecting and Characterizing Circular RNAs. Nature Biotechnology, 32, 453-461. https://doi.org/10.1038/nbt.2890

[7] Toptan, T., et al. (2018) Circular DNA Tumor Viruses Make Circular RNAs. Proceedings of the National Academy of Sciences of the United States of America, 115, E8737-E8745. https://doi.org/10.1073/pnas.1811728115

[8] Ungerleider, N., et al. (2018) The Epstein Barr Virus circRNAome. PLOS Pathogens, 14, e1007206. https://doi.org/10.1371/journal.ppat.1007206

[9] Jin, X., Feng, C.Y., Xiang, Z., Chen, Y.P. and Li, Y.M. (2016) CircRNA Expression Pattern and circRNA-miRNA-mRNA Network in the Pathogenesis of Nonalcoholic Steatohepatitis. Oncotarget, 7, 66455-66467.

https://doi.org/10.18632/oncotarget.12186

[10] Barrett, S.P. and Salzman, J. (2016) Circular RNAs: Analysis, Expression and Potential Functions. Development, 143, 1838-1847. https://doi.org/10.1242/dev.128074

[11] Lyu, D. and Huang, S. (2016) The Emerging Role and Clinical Implication of Human Exonic Circular RNA. RNA Biology, 14, 1000-1006.

[12] Salzman, J., Gawad, C., Wang, P.L., Lacayo, N. and Brown, P.O. (2012) Circular RNAs Are the Predominant Transcript Isoform from Hundreds of Human Genes in Diverse Cell Types. PLoS ONE, 7, e30733.

https://doi.org/10.1371/journal.pone.0030733

[13] Hsiao, K.Y., Sun, H.S. and Tsai, S.J. (2017) Circular RNA-New Member of Noncoding RNA with Novel Functions. Experimental Biology and Medicine, 242, 1136-1141. https://doi.org/10.1177/1535370217708978

[14] Sanger, H.L., Klotz, G., Riesner, D., Gross, H.J. and Kleinschmidt, A.K. (1976) Viroids Are Single-Stranded Covalently Closed Circular RNA Molecules Existing as Highly Base-Paired Rod-Like Structures. Proceedings of the National Academy of Sciences of the United States of America, 73, 3852-3856. https://doi.org/10.1073/pnas.73.11.3852

[15] Guo, J.U., Agarwal, V., Guo, H. and Bartel, D.P. (2014) Expanded Identification and Characterization of Mammalian Circular RNAs. Genome Biology, 15, 409. 
https://doi.org/10.1186/PREACCEPT-1176565312639289

[16] Jeck, W.R., et al. (2013) Circular RNAs Are Abundant, Conserved, and Associated with ALU Repeats. RNA, 19, 141-157. https://doi.org/10.1261/rna.035667.112

[17] Salzman, J., Chen, R.E., Olsen, M.N., Wang, P.L. and Brown, P.O. (2013) Cell-Type Specific Features of Circular RNA Expression. PLOS Genetics, 9, e1003777. https://doi.org/10.1371/journal.pgen.1003777

[18] Carninci, P., et al. (2005) The Transcriptional Landscape of the Mammalian Genome. Science, 309, 1559-1563. https://doi.org/10.1126/science.1112014

[19] Wang, M., et al. (2017) Circular RNAs: A Novel Type of Non-Coding RNA and Their Potential Implications in Antiviral Immunity. International Journal of Biological Sciences, 13, 1497-1506. https://doi.org/10.7150/ijbs.22531

[20] Zhao, Z.J. and Shen, J. (2017) Circular RNA Participates in the Carcinogenesis and the Malignant Behavior of Cancer. RNA Biology, 14, 514-521.

https://doi.org/10.1080/15476286.2015.1122162

[21] Pamudurti, N.R., et al. (2017) Translation of CircRNAs. Molecular Cell, 66, 9-21e7. https://doi.org/10.1016/j.molcel.2017.02.021

[22] Sand, M., et al. (2016) Circular RNA Expression in Cutaneous Squamous Cell Carcinoma. Journal of Dermatological Science, 83, 210-218.

https://doi.org/10.1016/j.jdermsci.2016.05.012

[23] Miao, R., et al. (2017) Microarray Expression Profile of Circular RNAs in Chronic Thromboembolic Pulmonary Hypertension. Medicine, 96, e7354. https://doi.org/10.1097/MD.0000000000007354

[24] Jiang, G., et al. (2017) Relationships of Circular RNA with Diabetes and Depression. Scientific Reports, 7, Article No. 7285. https://doi.org/10.1038/s41598-017-07931-0

[25] Haque, S. and Harries, L.W. (2017) Circular RNAs (circRNAs) in Health and Disease. Genes (Basel), 8, 353. https://doi.org/10.3390/genes8120353

[26] Chen, Y.G., et al. (2017) Sensing Self and Foreign Circular RNAs by Intron Identity. Molecular Cell, 67, 228-238e5. https://doi.org/10.1016/j.molcel.2017.05.022

[27] Li, X., et al. (2017) Coordinated circRNA Biogenesis and Function with NF90/NF110 in Viral Infection. Molecular Cell, 67, 214-227e7. https://doi.org/10.1016/j.molcel.2017.05.023

[28] Zhang, X., et al. (2017) Circular RNA Alterations Are Involved in Resistance to Avian Leukosis Virus Subgroup-J-Induced Tumor Formation in Chickens. Oncotarget, 8, 34961-34970. https://doi.org/10.18632/oncotarget.16442

[29] Legnini, I., et al. (2017) Circ-ZNF609 Is a Circular RNA That Can Be Translated and Functions in Myogenesis. Molecular Cell, 66, 22-37.e9.

https://doi.org/10.1016/j.molcel.2017.02.017

[30] Yang, Y., et al. (2017) Extensive Translation of Circular RNAs Driven by N6-Methyladenosine. Cell Research, 27, 626-641. https://doi.org/10.1038/cr.2017.31

[31] Wang, Y. and Wang, Z. (2015) Efficient Backsplicing Produces Translatable Circular mRNAs. $R N A, 21,172-179$. https://doi.org/10.1261/rna.048272.114

[32] Wei, C.M., Gershowitz, A. and Moss, B. (1975) Methylated Nucleotides Block 5' Terminus of HeLa Cell Messenger RNA. Cell, 4, 379-386. https://doi.org/10.1016/0092-8674(75)90158-0

[33] Li, S. and Mason, C.E. (2014) The Pivotal Regulatory Landscape of RNA Modifications. Annual Review of Genomics and Human Genetics, 15, 127-150. https://doi.org/10.1146/annurev-genom-090413-025405 
[34] Sun, C., Querol-Audi, J., Mortimer, S.A., et al. (2013) Two RNA-Binding Motifs in eIF3 Direct HCV IRES-Dependent Translation. Nucleic Acids Research, 41, 7512-7521. https://doi.org/10.1093/nar/gkt510

[35] Meyer, K.D., Patil, D.P., Zhou, J., et al. (2015) 5' UTR m(6)A Promotes Cap Independent Translation. Cell, 163, 999-1010.

https://doi.org/10.1016/j.cell.2015.10.012

[36] Liberman, N., Gandin, V., Svitkin, Y.V., et al. (2015) DAP5 Associates with eIF2beta and eIF4AI to Promote Internal Ribosome Entry Site Driven Translation. Nucleic Acids Research, 43, 3764-3775. https://doi.org/10.1093/nar/gkv205

[37] Zhang, Y., et al. (2017) Circular RNAs: Emerging Cancer Biomarkers and Targets. Journal of Experimental \& Clinical Cancer Research, 36, 152.

https://doi.org/10.1186/s13046-017-0624-Z

[38] Peng, L., Yuan, X.Q. and Li, G.C. (2015) The Emerging Landscape of Circular RNA ciRS-7 in Cancer (Review). Oncology Reports, 33, 2669-2674. https://doi.org/10.3892/or.2015.3904

[39] Hansen, T.B., Kjems, J. and Damgaard, C.K. (2013) Circular RNA and miR-7 in Cancer. Cancer Research, 73, 5609-5612. https://doi.org/10.1158/0008-5472.CAN-13-1568

[40] Fischer, J.W. and Leung, A.K. (2017) CircRNAs: A Regulator of Cellular Stress. Critical Reviews in Biochemistry and Molecular Biology, 52, 220-233. https://doi.org/10.1080/10409238.2016.1276882

[41] Du, W.W., et al. (2017) Foxo3 Circular RNA Promotes Cardiac Senescence by Modulating Multiple Factors Associated with Stress and Senescence Responses. European Heart Journal, 38, 1402-1412.

[42] Zheng, Q., et al. (2016) Circular RNA Profiling Reveals an Abundant circHIPK3 That Regulates Cell Growth by Sponging Multiple miRNAs. Nature Communications, 7, Article No. 11215. https://doi.org/10.1038/ncomms11215

[43] Wang, F., Nazarali, A.J. and Ji, S. (2016) Circular RNAs as Potential Biomarkers for Cancer Diagnosis and Therapy. American Journal of Cancer Research, 6, 1167-1176.

[44] Abdelmohsen, K., et al. (2017) Identification of HuR Target Circular RNAs Uncovers Suppression of PABPN1 Translation by circPABPN1. RNA Biology, 14, 361-369. https://doi.org/10.1080/15476286.2017.1279788

[45] Li, Z., et al. (2015) Exon Intron Circular RNAs Regulate Transcription in the Nucleus. Nature Structural \& Molecular Biology, 22, 256-264. https://doi.org/10.1038/nsmb.2959

[46] Ashwal-Fluss, R., Meyer, M., Pamudurti, N.R., Ivanov, A., Bartok, O. and Hanan, M. (2014) circRNA Biogenesis Competes with pre-mRNA Splicing. Molecular Cell, 56, 55-66. https://doi.org/10.1016/j.molcel.2014.08.019

[47] Conn, V.M., et al. (2017) A circRNA from SEPALLATA3 Regulates Splicing of Its Cognate mRNA through R-Loop Formation. Nature Plants, 3, 17053. https://doi.org/10.1038/nplants.2017.53

[48] Cadena, C. and Hur, S. (2017) Antiviral Immunity and Circular RNA: No End in Sight. Molecular Cell, 67, 163-164. https://doi.org/10.1016/j.molcel.2017.07.005

[49] Sun, L., Liu, S. and Chen, Z.J. (2010) SnapShot: Pathways of Antiviral Innate Immunity. Cell, 140, 436e2. https://doi.org/10.1016/j.cell.2010.01.041

[50] Kell, A.M. and Gale, M.J. (2015) RIG-I in RNA Virus Recognition. Virology, 479-480, 110-121. https://doi.org/10.1016/j.virol.2015.02.017 
[51] Cullen, B.R. (2006) Viruses and microRNAs. Nature Genetics, 38, S25-S30. https://doi.org/10.1038/ng1793

[52] Ghosh, Z., Mallick, B. and Chakrabarti, J. (2009) Cellular versus Viral microRNAs in Host-Virus Interaction. Nucleic Acids Research, 37, 1035-1048. https://doi.org/10.1093/nar/gkn1004

[53] Scaria, V., Hariharan, M., Maiti, S., Pillai, B. and Brahmachari, S.K. (2006) Host-Virus Interaction: A New Role for microRNAs. Retrovirology, 3, 1-9. https://doi.org/10.1186/1742-4690-3-68

[54] Skalsky, R.L. and Cullen, B.R. (2010) Viruses, microRNAs, and Host Interactions. Annual Review of Microbiology, 64, 123-141. https://doi.org/10.1146/annurev.micro.112408.134243

[55] Louten, J., Beach, M., Palermino, K., Weeks, M. and Holenstein, G. (2015) MicroRNAs Expressed during Viral Infection: Biomarker Potential and Therapeutic Considerations. Biomarker Insights, 10, 25-52. https://doi.org/10.4137/BMI.S29512

[56] Cai, X., et al. (2006) Epstein-Barr Virus microRNAs Are Evolutionarily Conserved and Differentially Expressed. PLOS Pathogens, 2, e23. https://doi.org/10.1371/journal.ppat.0020023

[57] Gartner, J.J., Sethupathy, P., Hatzigeorgiou, A.G. and Fraser, N.W. (2008) Anti Apoptotic Function of a microRNA Encoded by the HSV-1 Latency-Associated Transcript. Nature, 451, 600. https://doi.org/10.1038/nature06621

[58] Sullivan, C.S., Grundhoff, A.T., Tevethia, S., Pipas, J.M. and Ganem, D. (2005) SV40-Encoded microRNAs Regulate Viral Gene Expression and Reduce Susceptibility to Cytotoxic T Cells. Nature, 435, 682-686. https://doi.org/10.1038/nature03576

[59] Jung, Y.-J., Choi, H., Kim, H. and Lee, S.K. (2014) MicroRNA miR-BART20-5p Stabilizes Epstein-Barr Virus Latency by Directly Targeting BZLF1 and BRLF1. Journal of Virology, 88, 9027-9037. https://doi.org/10.1128/JVI.00721-14

[60] Qiu, J., Smith, P., Leahy, L. and Thorley-Lawson, D.A. (2015) The Epstein-Barr Virus Encoded BART miRNAs Potentiate Tumor Growth in Vivo. PLOS Pathogens, 11, e1004561. https://doi.org/10.1371/journal.ppat.1004561

[61] He, L., et al. (2017) Deep Circular RNA Sequencing Provides Insights into the Mechanism Underlying Grass Carp Reovirus Infection. International Journal of Molecular Sciences, 18, pii: E1977. https://doi.org/10.3390/ijms18091977

[62] Chen, J., et al. (2017) Circular RNA Profile Identifies circPVT1 as a Proliferative Factor and Prognostic Marker in Gastric Cancer. Cancer Letters, 388, 208-219. https://doi.org/10.1016/j.canlet.2016.12.006

[63] Suzuki, H. and Tsukahara, T.A. (2014) View of pre-mRNA Splicing from RNase R Resistant RNAs. International Journal of Molecular Sciences, 15, 9331-9342. https://doi.org/10.3390/ijms15069331

[64] Lasda, E. and Parker, R. (2016) Circular RNA Co-Precipitate with Extracellular Vesicles: A Possible Mechanism for circRNA Clearance. PLoS ONE, 11, e0148407. https://doi.org/10.1371/journal.pone.0148407

[65] Memczak, S., Papavasileiou, P., Peters, O. and Rajewsky, N. (2015) Identification and Characterization of Circular RNAs as a New Class of Putative Biomarkers in Human Blood. PLoS ONE, 10, e0141214. https://doi.org/10.1371/journal.pone.0141214

[66] Bahn, J.H., et al. (2014) The Landscape of microRNA, Piwi-Interacting RNA, and Circular RNA in Human Saliva. Clinical Chemistry, 61, 221-230. 
https://doi.org/10.1373/clinchem.2014.230433

[67] Shao, Y., et al. (2017) Global Circular RNA Expression Profile of Human Gastric Cancer and Its Clinical Significance. Cancer Medicine, 6, 1173-1180.

https://doi.org/10.1002/cam4.1055

[68] Li, Y., et al. (2015) Circular RNA Is Enriched and Stable in Exosomes: A Promising Biomarker for Cancer Diagnosis. Cell Research, 25, 981-984.

https://doi.org/10.1038/cr.2015.82

[69] Li, P., et al. (2015) Using Circular RNA as a Novel Type of Biomarker in the Screening of Gastric Cancer. Clinica Chimica Acta, 444, 132-136. https://doi.org/10.1016/j.cca.2015.02.018

[70] Chen, S., Li, T., Zhao, Q., Xiao, B. and Guo, J. (2017) Using Circular RNA Hsa_Circ_0000190 as a New Biomarker in the Diagnosis of Gastric Cancer. Clinica Chimica Acta, 466, 167-171. https://doi.org/10.1016/j.cca.2017.01.025

[71] Sun, Y.M., Lin, K.Y. and Chen, Y.Q. (2013) Diverse Functions of miR-125 Family in Different Cell Contexts. Journal of Hematology \& Oncology, 6, 6.

https://doi.org/10.1186/1756-8722-6-6 